\title{
Europäische Union oder Disunion?
}

Vor 60 Jahren wurde mit der Unterzeichnung der Römischen Verträge der wichtigste Grundstein der heutigen EU gelegt. Nach über 50 Jahren relativ stetiger Erweiterungs- und Vertiefungsschritte, die zur EU28 und - für mittlerweile 19 Mitgliedstaaten - auch zu einer gemeinsamen Währung führten, zeigen sich seit einigen Jahren allerdings auch klare gegenläufige Tendenzen: So wird 2009 durch das im Vertrag von Lissabon beschlossene Austrittsrecht zum ersten Mal auch eine Umkehrbarkeit des Integrationsprozesses offiziell anerkannt. Mit der Diskussion um einen möglichen Grexit werden Rückschritte bezüglich der Integrationstiefe - zumindest für einzelne Länder - zur Option erklärt. Selbst EZB-Präsident Draghi sprach jüngst die Möglichkeit eines Austritts aus der Eurozone an. Insgesamt beginnt die Bereitschaft, nationale Souveränität an die EU abzugeben, immer mehr zu schwinden, nationale Interessen konkurrieren zunehmend mit Gemeinschaftszielen, der Integrationsraum beginnt zu schrumpfen (Brexit). Hinzu kommen „Fliehkräfte“ durch nationalistische und rechtspopulistische Bewegungen in Frankreich, Italien, den Niederlanden, Dänemark, Ungarn und anderen Mitgliedsländern. Ein Austritt aus der Eurozone wird in diesen Ländern ebenso thematisiert wie ein möglicher Austritt aus der EU insgesamt. Nicht zuletzt die Flüchtlingskrise zeigt, dass die Bindewirkung demokratischer Wertvorstellungen innerhalb der EU nachlässt und eine Gemeinwohlorientierung in den Mitgliedsländern zunehmend schwindet, seit nicht mehr nur Wohltaten, sondern auch Lasten zu verteilen sind.

Auch das Eurobarometer spiegelt diese Entwicklungen: Bei der letzten Befragung im Herbst 2016 stimmten bei der Frage, ob die EU ein positives oder negatives Bild hervorrufe, nur 35\% der Befragten für positiv. Zugleich waren nur 23\% der Befragten der Meinung, dass sich die Dinge in der EU „derzeit in die richtige Richtung bewegen“, $56 \%$ dagegen hielten die eingeschlagene Richtung derzeit für falsch. Und bei der Vertrauensfrage sprachen aktuell nur 36\% der EU ihr Vertrauen aus, 54\% nicht. 2007 war Vertrauen in die EU dagegen noch bei $57 \%$ der Befragten vorhanden.

Was brachte nun den fortschreitenden Integrationsprozess ins Stocken? Die Problematik der EU besteht in dem Versuch, simultan Erweiterung und Vertiefung („eine immer engere Union“) voranzutreiben. Die sukzessive Vergrößerung der Gemeinschaft um periphere Länder erhöht naturgemäß die Heterogenität der Wirtschaftsstrukturen, der wirtschaftspolitischen Notwendigkeiten, der Präferenzen und Interessen. Hierdurch steigen die sogenannten „Entscheidungskosten“ bei den Gemeinschaftsaktivitäten: Je nach Entscheidungsregel entstehen Kosten entweder bei der überstimmten Minderheit (Kosten der Entscheidungsduldung bei Mehrheitsentscheidungen) oder bei der Mehrheit, wenn eine Entscheidung, die den meisten nützen würde, aufgrund des Vetos eines oder mehrerer Mitgliedsländer unterbleibt. Gleich, welche Entscheidungsmodalitäten die EU wählt, sind diese Entscheidungskosten umso gravierender, je heterogener die Präferenzen der Mitgliedsländer sind. Will man die Entscheidungskosten verringern, so müssen entweder weniger Entscheidungen getroffen werden - dies liefe auf eine geringere Integrationstiefe hinaus, oder die Entscheidungen müssen in homogeneren Gruppen getroffen werden - dies liefe auf eine kleinere Gemeinschaft bzw. auf kleinere Integrationsclubs innerhalb des Clubs EU hinaus.

Die Wechselbeziehungen zwischen den (vor allem ökonomischen) Vorteilen einer großen Gemeinschaft und den (vor allem politischen) Kosten der Heterogenität in einer solch großen Gemeinschaft sind seit langem bekannt (dokumentiert in verschiedenen Texte von Alberto Alesina): Für politische Stabilität ist die optimale Gemeinschaftsgröße kleiner als für wirtschaftliche Effizienz. Wirtschaftlich effiziente Integra-

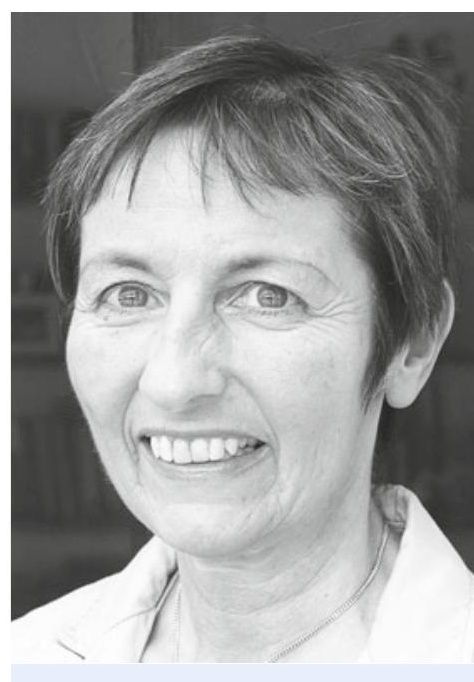

Renate Ohr ist Professorin für Volkswirtschaftslehre an der Wirtschaftswissenschaftlichen Fakultät der Georg-AugustUniversität Göttingen und Mitglied der Akademie der Wissenschaften zu Göttingen. 
tionsräume sind größer, hierdurch in sich heterogener und somit politisch instabiler. Will man aber wirtschaftliche Effizienz durch Skaleneffekte und politische Stabilität kombinieren, so müsste für die Integrationsstufe der Marktintegration (Binnenmarkt) ein großer Integrationsraum angestrebt werden, während für politisch/institutionelle Integration - der Vergemeinschaftung institutioneller Regelungen, Eingriffe und Politiken, wie bei der Währungsunion - kleinere, homogenere Integrationsräume gebildet werden müssten.

Die Globalisierung der Märkte schwächt allerdings die komparativen Vorteile regionaler Freihandelsgemeinschaften und damit die Binnenmarkt-Vorteile eines großen regionalen Integrationsraums. Die Globalisierung ermöglicht mittlerweile, dass weitgehender Freihandel auch außerhalb der EU und damit ohne die Kosten der institutionellen Mitgliedschaft in einer so großen Gemeinschaft sehr unterschiedlicher Länder möglich ist. Bei unveränderter Heterogenität in der Union kann hierdurch Desintegration gefördert werden, wenn die Instabilitäten und Kosten der institutionellen Integration die - nun relativierten - Vorteile der Marktintegration überkompensieren.

Ist nun eine immer engere (Rest-)Union die Lösung? Die Europäische Union ist eine weltweit einmalige Erfolgsgeschichte, die es zu erhalten gilt! Die Integrationsbereitschaft darf jedoch nicht überschätzt und nicht überfordert werden. Integration, Solidarität, gemeinsame Wertvorstellungen und Präferenzen sowie gemeinsames Handeln kann man nicht erzwingen. Durch überzogene Forderungen nach einer immer engeren Union setzt man die bisherigen Integrationserfolge aufs Spiel. Stattdessen ist eine neue identitätsstiftende Vision nötig. Als einigende Klammer der EU kann dabei die Grundidee des Binnenmarktes betrachtet werden. Selbst die in Großbritannien kritisierte Binnenmarkt-Grundfreiheit der Freizügigkeit erscheint laut Eurobarometer über $80 \%$ der EU-Bürger als wünschenswert. „Bruchstellen“ sind der Euro und die fehlende Gemeinwohlorientierung, die für eine stärkere politische Integration notwendig wäre.

Eine Auflösung des Konflikts zwischen der Größe des Integrationsraums und der Integrationstiefe bietet daher die schon in den 1990er Jahren diskutierte größere Integrationsflexibilität, etwa in Form eines „Europas der konzentrischen Kreise“. Hier würde ein (homogeneres) „Kerneuropa“ eine relativ große Integrationstiefe erreichen, andere Mitgliedsländer der EU könnten sich jedoch - temporär oder dauerhaft - auf weniger Gemeinsamkeiten beschränken. Entscheidend ist es, jene Bereiche zu identifizieren, an denen sich alle beteiligen müssen, und jene Bereiche, für die eine flexible Teilnahme möglich sein soll.

Vor dem Hintergrund der schon bestehenden monetären Integration muss zugleich eine tragfähige und langfristig stabile Lösung für die Probleme der Eurozone gefunden werden, um eine Übertragung der Instabilitäten dort auf die gesamte Europäische Union zu vermeiden. Dazu kann auch das Ausscheiden einzelner Länder aus der Eurozone - nicht aus der EU! - gehören. Dem Festhalten an einer unveränderten Eurozone darf kein höherer Wert beigemessen werden als der nachhaltigen Sicherung der EU insgesamt. Eine flexible Integrationsstrategie würde es den Mitgliedsländern der EU ermöglichen, weiterhin die Vorteile eines großen gemeinsamen Binnenmarktes zu nutzen, aber z.B. die Probleme einer gemeinsamen Geld- und Währungspolitik in der sehr heterogenen Eurozone zu vermeiden, indem diese Form institutioneller Integration auf einen kleineren, homogeneren Konvergenzclub reduziert werden könnte. Mehr Integrationsflexibilität und mehr Konzentration auf Marktintegration statt auf institutionelle Integration müssen künftig im Vordergrund stehen, um zu verhindern, dass die aktuellen Strömungen aus der Europäischen Union 